\title{
喿Iresalce
}

\section{Tecnologias educacionais como estratégia para educação em saúde de adolescentes: revisão integrativa}

\author{
Educational technologies as strategy for adolescent health education: Integrative review
}

Mikaelle Ysis da Silva1, Danielle Elias Gonçalves², Álissan Karine Lima Martins ${ }^{3}$

\begin{abstract}
Resumo
Introdução: na adolescência, fase que envolve diversas mudanças e requer específica atenção, torna-se relevante o uso de tecnologias educacionais junto ao processo de educação em saúde, como meio eficaz para o alcance da promoção da saúde. Objetivo: identificar, na literatura científica, tecnologias educacionais desenvolvidas, validadas e/ou aplicadas, com ênfase na educação em saúde de adolescentes. Método: revisão integrativa, realizada através do Portal de Periódicos da Coordenação de Aperfeiçoamento de Pessoal de Nível Superior, por meio das bases de dados MEDLINE, LILACS, IBECS, BDENF e CINAHL, utilizando-se dos descritores: Adolescente, Tecnologia Educacional e Educação em Saúde. Resultados: a amostra final quantificou 34 estudos, os quais apresentam bons níveis de evidência científica e destacam-se pela diversidade entre as tecnologias educacionais do tipo virtual e impresso, como site, website, cybertutor, bate papo, webnovela, plataformas digitais, programas de ensino, jogos, dramatizações, flipcharts, vídeos, histórias em quadrinhos, fotonovela e livro informativo. A principal ênfase a qual se volta às condutas de educação em saúde esta vinculada à saúde sexual e reprodutiva. Considerações finais: o estudo apresenta contribuições relevantes para $o$ campo da saúde, em especial às profissões que buscam modalidade de ações inovadoras para incremento das práticas de promoção da saúde direcionadas a adolescentes.
\end{abstract}

Palavras-chave: Adolescente, Tecnologia Educacional e Educação em Saúde.

\begin{abstract}
Introduction: In adolescence, phase that involves several changes and requires specific attention, becomes relevant or the use of educational technologies in the health education process, as an effective means to achieve health promotion. Objective: to identify in the scientific literature, educational technologies carried out and validated in health education for adolescents. Method: integrative review mostly accomplished by Portal de Periódicos da Coordenação de Aperfeiçoamento de Pessoal de Nível Superior available in the databases MEDLINE, LILACS, IBECS, BDENF and CINAHL and, through the descriptors "adolescent", "technology educational", "health education". Results: the final sample identified 34 studies that demonstrates good levels of scientific evidence and stand out for diversity in the use of ETs virtual and printed, such as, sites, websites, online chat, web novels, digital platforms, online courses, games, dramatize, flipcharts, videos, comic books, romance novel, informative books. The main themes addressed refer to the health education to promote
\end{abstract}

1 Especialista. Universidade Regional do Cariri (URCA). Email: mikaelleysis02@gmail.com

2 Graduada. Universidade Regional do Cariri (URCA).E-mail: danielleelias_golcalves@hotmail.com

3 Doutora. Universidade Regional do Cariri (URCA). E-mail: alissan.martins@urca.br

Correspondência: Rua Coronel Antônio Luíz, 1161 - Pimenta, Crato - CE, 63105-010.

Rev. Saúde Digital Tec. Educ., Fortaleza, CE, v. 5, n. 1, p.66-82, jan./abr. 2020. ISSN: 2525-9563 
sexual and reproductive health. Final considerations: the study presents relevant contributions for health education, mostly the professionals looking for innovations to promote adolescent health.

Keywords: Adolescent, Educational Technology e Health Education.

\section{Introdução}

No Brasil, de acordo com o Estatuto da Criança e do Adolescente (ECA), o período da adolescência compreende a idade de doze a dezenove anos ${ }^{1}$. Caracteriza-se como fase socialmente construída, com destaque para necessidade de considerar as diferentes realidades locais ${ }^{2}$ como meio para propiciar o direcionamento da assistência à saúde, que apresenta limitações, diante da ausência de práticas específicas ao cuidado ${ }^{3}$.

Torna-se evidente a necessidade de mudanças no contexto da assistência à saúde, requerendo maior atenção a essa população, diante das vulnerabilidades em que esta encontra-se exposta, em decorrência do processo de transição para vida adulta. Assim, faz-se necessário o acesso à informação de qualidade, de modo que oportunize maior autonomia aos sujeitos ${ }^{4,5}$.

Nesse sentido, as Tecnologias Educacionais (TE) devem ser vistas enquanto meios que proporcionem as atividades de educação em saúde, como materiais de ensino que dinamizam as ações $^{6}$. O uso da TE possibilitam que os momentos de educação em saúde tornemse mais diversos, sendo quesito essencial para o alcance da aprendizagem ${ }^{7}$.
Desta forma, elaborou-se a seguinte questão norteadora: Quais TE estão sendo desenvolvidas, validadas e/ou aplicadas com ênfase na educação em saúde de adolescentes?

Portanto, objetivou-se identificar na literatura científica as TE desenvolvidas, validadas e/ou aplicadas com ênfase na educação em saúde do público adolescente.

Desse modo, este estudo torna-se relevante, por identificar a utilização das TE em saúde direcionada ao público adolescente, incentivando outros estudos na construção, validação ou até mesmo no aprimoramento de tecnologias educacionais existentes, como também práticas de educação em saúde direcionada a adolescentes.

\section{Métodos}

Trata-se de Revisão Integrativa (Rl) que compreende fonte de dados secundária da literatura, delimitada através de método de busca rigoroso. A enfermagem empodera-se da utilização da $\mathrm{RI}$, por acreditar ser prática capaz de corresponder às necessidades de pesquisa, amparada no fortalecimento das Pesquisas Baseadas em Evidências $(\mathrm{PBE})^{8}$. 
Para o desenvolvimento da $\mathrm{Rl}$, seguiram-se criteriosamente as seis etapas: estabelecimento do problema de pesquisa; buscas dos estudos na literatura, através das bases de dados, por meio dos critérios de inclusão e exclusão; categorização dos estudos para extração das informações; análise crítica dos estudos; interpretação dos resultados; e compilado geral e/ou apresentação ${ }^{9}$.

A delimitação do problema de pesquisa ocorreu através da estratégia de pesquisa Population, Variables and Outcomes (PVO), delimitando os descritores de busca, sendo os Descritores em Ciências da Saúde (Decs) e os Medical Subject Headings (MeSH) (Figura 1- nos anexos).

De março a julho de 2018, buscaram-se os estudos de forma pareada, através dos referidos Decs e MeSh, utilizando-se do operador boleando AND. O acesso aos estudos deu-se através do Portal de Periódicos da Coordenação de Aperfeiçoamento de Pessoal de Nível Superior (CAPES), selecionaram-se as bases de dados conforme o escopo de abrangência e a representatividade, quais sejam: Medical Literature Analysis and Retrieval System Online (MEDLINE), Literatura Latinoamericana e do Caribe em Ciências da Saúde (LILACS), Índice Bibliográfico Español em Ciencias de la Salud (IBECS), Base de Dados em Enfermagem (BDENF) e Cumulative Index to Nursing and Allied Health Literature (CINAHL). Destaca-se que em cada base de dados foram realizados os seguintes cruzamentos: cruzamento 1: "Adolescente AND Tecnologia Educacional”; cruzamento 2 "Adolescente AND Educação em Saúde"; e cruzamento 3: "Tecnologia educacional AND Educação em Saúde".

Para filtrar os estudos, conforme a ênfase do fenômeno investigado, empregaram-se os critérios de inclusão: estudos originais, disponíveis em formato completo, com abordagem qualitativa ou quantitativa; estudos que tivessem relação direta com o objeto de estudo, os quais deveriam envolver adolescentes, tecnologia educacional e educação em saúde e artigos publicados nos idiomas português, inglês ou espanhol. Os critérios de exclusão foram: estudos com data de publicação anterior aos últimos cinco anos (2014 a 2018), diante do critério de atualidade; estudos repetidos, revisões, relatos de experiência, teses, dissertações ou editoriais, e estudos cujos métodos não estivessem rigorosamente definidos. A seleção foi realizada através do fluxograma Preferred Reporting Items for Systematic Review and Meta-Analyses PRISMA $^{10}$ (Figura 2 - nos anexos).

Para análise dos estudos, aplicou-se instrumento de elaboração própria, com inclusão dos itens: autores/país/ano; tipo de estudo e nível de evidência; TE utilizada (desenvolvida, aplicada, avaliada e/ou validada) e as implicações para a prática (Quadro 1 - nos anexos). 
Os níveis de evidência foram quantificados conforme os níveis descritos ${ }^{11}$ : nível 1 (revisões sistemáticas e metanálises de ensaios clínicos randomizados), nível 2 (ensaio clínico controlado randomizado), nível 3 (ensaio clínico sem randomização), nível 4 (estudo de coorte e caso controle), nível 5 (revisões sistemáticas de estudos descritivos e qualitativos), nível 6 (estudos descritivos ou qualitativos) e nível 7 (estudos que advém da opinião de autoridades ou especialistas).

\section{Resultados}

A amostra final quantificou 34 estudos, destes, 20 da CINAHL, nove da MEDLINE, três da BDENF e dois da LILACS (Quadro 1 - nos anexos).

Observou-se número considerável de publicações recentes acerca da construção, validação ou aplicabilidade de TE voltadas à educação em saúde dos adolescentes.

Com ênfase ao país de publicação, em maioria, os estudos foram publicados nos EUA $(26,47 \%)^{12-20}$, seguidos das publicações no Brasil $(17,65 \%)^{21-26}$ e na Índia $\quad(20,59 \%)^{27-33}$. Constataram-se estudos publicados na Austrália $(5,88 \%)^{34,35}$, Holanda $(5,88 \%)^{36,37}$, Cingapura $(2,94 \%)^{38}$, Espanha $(2,94 \%)^{39}$, Irlanda $(2,94 \%)^{40}$, França $(2,94 \%)^{41}$, México $(2,94 \%)^{42}$, Quênia $(2,94 \%)^{43}$, Romênia $(2,94 \%)^{44}$ e Tailândia $(2,94 \%)^{45}$.
Em relação ao ano de publicação, $29,41 \%$ foram publicados no ano de $2016^{14-16,24,29,30,34,37,41,44} ; 23,53 \%$, no ano de $2014^{19,20,26,32,33,35,43,45} ; 17,65 \%$ no ano de $2015^{17,18,25,31,39,40 ;} 17,65 \%$ no ano de $2017^{21,27,28,36,38,42}$; e $11,76 \%$ no ano de 2018 12,13,22,23, podendo haver menor quantitativo neste ano em específico, diante da não finalização do mesmo no período de seleção dos estudos.

Quanto ao nível de evidência dos estudos, $52,94 \%$ foram classificados com nível seis ${ }^{16-19,21,22,24-28,36,37,40-43,45} ; 23,53 \%$, nível três ${ }^{23,29-33,39,44}$; e 23,53\%, nível dois ${ }^{12-}$ $15,20,34,35,38$

Dentre os tipos de TE, identificaramse programas on-line, como site, website, cybertutor, bate papo, webnovela e plataformas digitais ${ }^{12,16-18,26,34,36,38,44,45}$, programas educacionais ${ }^{13-15,27-31,33,42}$, jogos ${ }^{21,22-24,25,37,41,43}$, vídeos ${ }^{30,40}$, dramatizações ${ }^{38}$, histórias em quadrinhos $^{19}$, mensagens enviadas pelo telefone ${ }^{20,35}$, ações educativas realizadas por rede social $^{23}$, fotonovela ${ }^{39}$ e livro informativo ${ }^{32}$.

Identificou-se que em $61,76 \%$ dos estudos, as TE estiveram voltadas à promoção da saúde sexual e/ou reprodutiva $^{12-17,19-22,24,30,31,33,34,36-40,43}$, seguida da educação nutricional $(14,71 \%)^{18,23,32,35,41}$, prevenção do uso do álcool e outras drogas $(11,76 \%)^{17,29,44,45}$, amamentação $\quad(2,94 \%)^{21}$, higiene $(2,94 \%)^{27}$, câncer $(5,88 \%)^{28,42}$, pessoa com deficiência $(2,94 \%)^{25}$, violência $(2,94 \%)^{17} \mathrm{e}$ síndromes genéticas $(2,94 \%)^{26}$. 
Em relação ao objetivo do estudo, a maioria das pesquisas (67,65\%) avaliou a eficácia de TE validadas ${ }^{12-16,20,23,25,27-39,42,44}$, seguidas de $17,65 \%$ que desenvolveram e aplicaram as TE, mas não validaram ${ }^{17,18,26,40,43,45}$. Enquanto que $5,88 \%$ apenas descreveram como deveria ser aplicada a $\mathrm{TE}^{19,24}$, outros 5,88\% efetivaram a validação e aplicação das tecnologias educacionais com o público adolescente $^{21,22}$. Identificou-se, ainda, estudo que realizou melhoria do conteúdo de TE construída e validada ${ }^{41}$.

Quando ao uso das TE e respectivas implicações para prática, $88,24 \%$ dos estudos apresentaram impacto positivo $^{12,13,15-22,24-33,36-45}$ e $11,76 \%$ não apresentaram qualquer tipo de efeito ${ }^{14,23,34,35}$.

\section{Discussões}

Diante dos pressupostos, as discussões estão apresentadas em duas categorias definidas conforme os principais resultados identificados, que incubem as tecnologias educacionais e os processos de educação em saúde.

\section{Tecnologias educacionais direcionadas ao público adolescente e implicações para prática}

As TE podem ser consideradas ferramentas eficazes e protagonistas no processo de consolidação da autonomia das pessoas, com enfoque principalmente no público adolescente ${ }^{46}$.
Diante dos benefícios, no contexto da educação em saúde dos adolescentes, através do uso das TE, torna-se promissor o desenvolvimento de estratégias que visem construção e validação de materiais educativos voltados a este público. Estudos afirmam e enfatizam essa necessidade diante da possibilidade de estímulo da autonomia e tomada de decisão pelos adolescentes ${ }^{22,47}$.

Ao tratar deste público, torna-se necessário que sejam implantadas metodologias que motivem o estabelecimento de relação educativa mais eficaz, como TE que estimulem experiências de vida saudáveis, por meio do protagonismo dos jovens, a partir do incentivo à participação autônoma no processo educativo ${ }^{48}$.

Os estudos da revisão buscaram promover a saúde dos adolescentes, através de materiais educativos, do tipo virtual e impresso.

Os materiais educativos de cunho impresso e/ou virtual podem ser complementares e um não anula o outro.

O material do tipo virtual caracterizase pela mídia eletrônica, apresenta relevância no quesito de interatividade ${ }^{49}$. 0 material do tipo impresso tem característica imutável, por conter atributos intrínsecos, após a impressão, este somente poderá ser atualizado através de outras edições ou impressões, contendo em cada uma das publicações aspectos únicos ${ }^{50}$. 
No estudo que investigou as práticas de ler textos no papel e na tela, sob a visão de graduandos do Bacharelado em Ciências e Tecnologia da Universidade Federal, do Rio Grande do Norte, em um dos relatos foi evidenciado que a leitura é estabelecida a partir da apreensão física do leitor pelo objeto, ao tipo de material impresso ${ }^{51}$.

Há carência na produção de materiais educativos impressos que potencializem a autonomia de indivíduos inclusos no contexto de populações de risco, sendo evidente a necessidade de ser estudada a melhor forma de como se apresentam esses materiais ${ }^{52}$.

Enfatiza-se a relevância do processo de desenvolvimento dos materiais, quanto ao envolvimento de profissionais capacitados na etapa de elaboração e validação destes ${ }^{52}$.

$O$ incremento de TE, junto aos adolescentes, é um método de estímulo para desenvolvimento da autonomia, primando pela melhor aquisição e apropriação do conhecimento, com foco nos assuntos abordados que, em maioria, envolvem contextos do processo de transição contida na fase da adolescência ${ }^{47}$.

Propor processos de educação em saúde, através da utilização das TE, é uma maneira de estímulo à integração e participação efetiva dos adolescentes junto às atividades desenvolvidas, em que estes apresentam maior interesse pelas temáticas abordadas, através do lúdico e da dinamicidade ${ }^{47}$.

Questionam-se as implicações práticas do uso das TE direcionadas ao público adolescente que, na presente revisão, pôde-se vislumbrar esse aspecto. Os estudos, em maioria, demostraram impactos positivos.

Nesse contexto, identificaram-se os seguintes quesitos como impactos positivos: confiabilidade das TE utilizadas; opção alternativa para o desenvolvimento de ações educativas; viabilidade; eficácia; caráter interativo; possibilidade de entretenimento; meio para aquisição de conhecimento; criatividade; diversidade; pensamento crítico-reflexivo; possibilidade de promover mudanças e aquisição de comportamentos positivos em saúde. Sendo estes impactos relacionados às TE do tipo: jogos educativos, programas online, programas de ensino, dramatizações, flipcharts, vídeos, programas de multimídia, fotonovela, webnovela, livro informativo, histórias em quadrinhos e

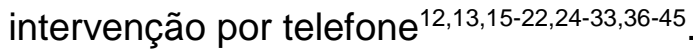

Outros estudos não identificaram impactos positivos na aplicabilidade das tecnologias, demonstraram poucas evidências do uso da tecnologia para apoiar a eficácia na prática, não atingiram o objetivo proposto e relataram que não obtiveram resultados quanto à aquisição de efeitos significativos, os quais referemse à ação educativa realizada pelo facebook, ao programa de ensino com foco na saúde sexual, ao programa virtual com 
ênfase na prevenção da gravidez na adolescência e intervenção realizada por smartphone, quanto à saúde nutricional ${ }^{14,23,34,35}$.

\section{Educação em saúde de adolescentes através das tecnologias educacionais}

A principal ênfase a qual se voltam às condutas de educação em saúde na presente revisão esteve vinculada à saúde sexual e/ou reprodutiva. A experiência e compreensão da sexualidade na adolescência tem sido alvo constante de estudos a nível nacional e internacional. Este é um componente inerente ao ser humano, a abordagem deste é fundamental na promoção da saúde integral de adolescentes e jovens ${ }^{22}$.

Nesse contexto, as TE estão vinculadas a busca dos adolescentes pelos recursos tecnológicos para aquisição de informações acerca da saúde, especialmente no tocante a assuntos que são tidos como tabu, como a sexualidade e a puberdade ${ }^{5}$.

A abordagem à educação alimentar também se destacou entre os estudos selecionados, como desenvolvimento de estudo que testou a melhoria do conhecimento sobre nutrição saudável em adolescentes e analisou o prazer dos participantes entre o jogo na internet "Gustavo in Gnam's Planet", em comparação com um jogo na web de lazer ${ }^{41}$.

A promoção de um estilo de vida saudável é uma questão de relevância social, de saúde e economia ${ }^{41}$. No entanto, existem questões que causam desmotivação, como a pouca disponibilidade de tempo para participar dos programas de promoção da saúde e das intervenções, além do baixo alcance do público-alvo, as quais, muitas vezes, tornam-se obstáculos para adoção e manutenção de uma vida saudável ${ }^{41}$.

As TE podem ser mais eficazes na produção de melhoria e sensibilização da importância do estilo de vida saudável, na medida em que incentiva o engajamento ativo, processamento de informações e aprendizado por diversão ${ }^{41}$.

As TE identificadas, também, voltamse para amamentação, higiene, câncer de boca, câncer de mama, pessoa com deficiência, violência e síndromes genéticas.

Além das áreas enfatizadas, também, evidenciaram-se tecnologias que abordavam a prevenção do uso do álcool e outras drogas ${ }^{17,29,44,45}$. Em estudo em que foram desenvolvidas histórias em quadrinhos com enfoque ao álcool, as autoras esclareceram que a temática na adolescência é pouco abordada em materiais educativos ou estão apresentadas juntamente com outros assuntos, como a droga ${ }^{53}$.

Em específico, no tocante à temática da prevenção ao uso do álcool e outras drogas, observa-se a utilização das TE como estratégia para sensibilizar os adolescentes acerca dos malefícios ocasionados por este ato, que pode 
comprometer o crescimento pessoal, profissional e, consequentemente, a qualidade de vida desses.

Dessa forma, reconhece-se a importância da abordagem de diferentes áreas, no que tange à educação em saúde dos adolescentes, visto que este é um público atrelado a diversas mudanças e em transição para vida adulta, sendo necessária abordagem integral, sem a escolha de um assunto em detrimento de outro. Para isso, deve-se ter o apoio de ferramentas específicas e direcionadas, atrativas, com linguagem adequada e condizente com a realidade.

\section{Conclusão}

A partir dos resultados obtidos por meio desta revisão integrativa, foi possível desvelar que o desenvolvimento de TE apresentam-se como estratégia para 0 incremento de ações em saúde destinadas aos adolescentes, cujo uso destas atrelase à potencialidade de benefícios, por compreender um tipo de material dialógico e que, em essência, é lúdico.

Nos últimos anos, são consideráveis as publicações científicas que destacam o desenvolvimento de TE junto ao público adolescente, no processo de educação em saúde, sendo estas diversas, englobando matérias do tipo virtual e impresso. Não se limitam a determinados tipos, estão abertas a diversas modalidades e campos de atuação. Aponta-se que um único tipo de material educativo pode ser utilizado em diferentes campos de atuação, desde que estejam adequados aos objetivos que se pretende atingir.

Pôde-se vislumbrar maioria significativa de estudos em que as TE apresentaram impactos positivos, podendo corroborar significativamente para aquisição de benefícios quanto às questões de saúde dos adolescentes.

Enfatiza-se a relevância da participação direta do público-alvo e dos profissionais da saúde junto ao processo de construção e validação desses materiais, para que assim possam atingir eficácia significativa e contribuir sobremaneira, junto à promoção da saúde dos adolescentes.

O estudo apresenta contribuições relevantes para o campo da saúde, diante da possibilidade de incrementar e inovar as práticas de educação em saúde, junto aos adolescentes, através do uso de TE identificadas, as quais envolvem diferentes contextos. Ademais, viabiliza-se aos profissionais da saúde modalidade de ações inovadoras para o incremento das práticas de educação em saúde direcionada aos adolescentes.

Todavia, acrescenta-se que o estudo apresentou limitações, estas relacionadas à indisponibilidade de alguns estudos, seja pelas limitações de indexação em bases de dados e/ou do acesso gratuito.

\section{Referências}

1. Brasil. Ministério da Saúde. Secretaria de Atenção à Saúde. Departamento de 
Ações Programáticas Estratégicas.

Política Nacional de Atenção Integral à

Saúde da Criança: orientações para implementação. Brasília; 2018.

2. Jager ME, Batista FA, Perrone CM, Santos SS, Dias ACG. O adolescente no contexto da saúde pública brasileira: reflexões sobre o PROSAD. Psicologia em Estudo, Maringá. 2014; 19 (2): 211 221. doi: 10.1590/1413-737221567004 3. Silva JF, Matsukura TS, Ferigato SH, Cid MFB. Adolescence and mental health from the perspective of Primary Healthcare professionals. Interface (Botucatu). 2019; 23: e180630. doi:

10.1590/Interface. 180630

4. Cavalcante RB, Ferreira MN, Maia LLQGN, Araújo A, Silveira RCP. Uso de Tecnologias da Informação e

Comunicação na educação em saúde de adolescentes escolares. J. Health Inform. 2012 [citado em 20185 de julho]; 4 (4): 182-186. Available from: http://www.jhisbis.saude.ws/ojs-jhi/index.php/jhisbis/article/view/197/142

5. Pinto ACS, Scopacasa LF, Bezerra LLAL, Pedrosa JV, Pinheiro PNC. Uso de tecnologias da informação e comunicação na educação em saúde de adolescentes: revisão integrativa. Rev enferm UFPE On line. 2017; 11(2):634-44. doi: 10.5205/reuol.10263-91568-1-

RV.1102201719.

6. Áfio ACE, Balbino AC, Alves MDS, Carvalho LV, Santos MCL, Oliveira NR.

Análise do conceito de tecnologia educacional em enfermagem aplicada ao paciente. Rev Rene. 2014 [citado em 201812 de ago]; 15(1):158-165. Available from:

http://www.periodicos.ufc.br/rene/article/vi ew/3108

7. Farias MS, Ponte KMA, Gomes DF, Menezes RSP. Tecnologia educativa sobre câncer gástrico. Rev enferm UFPE On line. 2018;12(4):947-952. doi: 10.5205/1981-8963-v12i4a230434p947952-2018.
8. Soares CB, Hoga LAK, Peduzzi M, Sangaleti C, Yonekura T, Silva DRAD. Revisão integrativa: conceitos e métodos utilizados na enfermagem. Rev Esc Enferm USP. 2014; 48(2):335-345. doi: 10.1590/S0080-6234201400002000020. 9. Mendes KDS, Silveira RCCP, Galvão $\mathrm{CM}$. Integrative literature review: a research method to incorporate evidence in health care and nursing. Texto Contexto Enferm. 2008; 17(4):758-764. doi: 10.1590/S0104-07072008000400018. 10. Moher D, Liberati A, Tetzlaff J, Altman DG. Preferred reporting items for systematic reviews and meta-analyses: the PRISMA statement. Int J Surg. 2009; 8(5):336-41. doi:

10.1371/journal.pmed.1000097.

11. Melnyk BM, Fineout-Overholt E. Making the case for evidence-based practice. In: Melnyk BM, Fineout-Overholt E. Evidence-based practice in nursing \& healthcare. A guide to best practice.

Philadelphia: Lippincot Williams \& Wilkins. 2005; p. 3-24.

12. Widman L, Golin CE, Kamke K, Burnette JL, Prinstein MJ. Sexual

Assertiveness Skills and Sexual DecisionMaking in Adolescent Girls: Randomized Controlled Trial of an Online Program. AJPH Research. 2018; 108(1):92-102. doi: 10.2105/AJPH.2017.304106. 13. Oman RF, Vesely SK, Green J, Clements-Nolle K, LU M. Adolescent Pregnancy Prevention Among Youths Living in Group Care Homes: A Cluster Randomized Controlled Trial. AJPH Research. 2018; 108 (Supl.1):538-544. doi: 10.2105/AJPH.2017.304126. 14. Francis $\mathrm{K}$, Philliber $\mathrm{S}$, Walsh-Buhi ER, Philliber A, Seshadri R, Daley E. Scalability of an Evidence-Based Adolescent Pregnancy Prevention Program: New Evidence From 5 ClusterRandomized Evaluations of the Teen Outreach Program. AJPH Research. 2016 [citado em 201822 de jun]; 106 (Supl. 1):532-538. Available from:

https://www.ncbi.nlm.nih.gov/pmc/articles/ PMC5049463/

15. Calise TV, Chow W, Doré KF, O'Brien MJ, Heitz ER, Millock RR. Healthy Futures Program and Adolescent Sexual Behaviors in 3 Massachusetts Cities: A Randomized Controlled Trial. AJPH 
research. 2016; 106 (Supl.1):103-109. doi: 10.2105/AJPH.2016.303389.

16. Wang $H$, Singhal A. East Los

High:Transmedia Edutainment to Promote the Sexual and Reproductive Health of Young Latina/o Americans. Perspectives From The Social Sciences. 2016; 106(6):1002-1010. doi:

10.2105/AJPH.2016.303072.

17. Andrade EL, Evans WD, Edberg MC, Cleary SD, Villalba R, Batista IC. Victor and Erika Webnovela: An Innovative Generation@ @udience Engagement Strategy for Prevention. J Health Commun. 2015; 20(12):1465-1472. doi: 10.1080/10810730.2015.1018648. 18. Kohlstadt IC, Steeves ETA, Rice K, Gittelsohn J, Summerfield LM, Gadhoke $P$. Youth peers put the "invent" into NutriBee's online intervention. Nutrition Journal. 2015; 14(60):1-8. doi: https://doi.org/10.1186/s12937-015-00312.

19. Katz ML, Oldach BR, Goodwin J, Reiter PL, Ruffin IV MT, Paskett ED. Development and Initial Feedback About a Human Papillomavirus (HPV) Vaccine Comic Book for Adolescents. J Canc Educ. 2014; 29:318-324. doi: 10.1007/s13187-013-0604-8.

20. DiClemente RJ, Wingood GM, Sales JM, Brown JL, Rose ES, Davis TL, Lang DL, Caliendo A, Hardin JW. Efficacy of a Telephone-Delivered Sexually Transmitted Infection/Human Immunodeficiency Virus Prevention Maintenance Intervention for Adolescents: A Randomized Clinical Trial. JAMA Pediatr. 2014; 168(10):938-946. doi: 10.1001/jamapediatrics.2014.1436. 21. Silva AKC, Oliveira KMM, Coelho MMF, Moura DJM, Miranda KCL. Construção e validação de jogo educativo para adolescentes sobre amamentação. Revista baiana de enfermagem. 2017;31(1):1-10. doi: $10.18471 /$ rbe.v31i1.16476. 22. Sousa MG, Oliveira EML, Coelho MMF, Miranda KCL, Henriques ACPT, Cabral RL. Validação de jogo educativo sobre sexualidade para adolescentes. J. res.: fundam. care. [online]. 2018; 10(1):203-209. doi: 10.9789/21755361.2018.v10i1.203-209.
23. Gonçalves RJM, Castro RAS, Belo VS, Coelho LSVA, Lagares EB, Novais RLR, Bila WC, Romano MCC. Online health: impact on the prevalence of obesity and body satisfaction of adolescents. J Nurs UFPE On line. 2018; 12(2):312-319. doi: 10.5205/1981-8963v12i2a230510p312-319-2018.

24. Souza V, Gazzinelli MF, Soares NA, Fernandes MM, Oliveira RNG, Fonseca RMGS. O jogo como estratégia para abordagem da sexualidade com adolescentes: reflexões teóricometodológicas. Rev Bras Enferm. 2017; 70(2):394-401. doi: 10.1590/0034-71672016-0043.

25. Silva JM, Pagliuc LMF, Carvalho AT, Oliveira MG, Almeida PC. Conhecimento de escolares acerca de pessoa com deficiência: jogo de labirinto na promoção da saúde. Rev enferm UERJ, Rio de Janeiro. 2015; 23(2):254-259. doi: 10.12957/reuerj.2015.10611. 26. Maximino LP, Picolini-Pereira MM, Carvalho JLB. Telegenetics: application of a tele-education program in genetic syndromes for Brazilian students. J Appl Oral Sci. 2014; 22(6):477-483. doi: 10.1590/1678-775720130311.

27. Savita G, Ranjitha D'S. To Assess the Effectiveness of Planned Teaching Programme on Knowledge and Practices Regarding Menstrual Hygiene among the Adolescent Girls in Selected Govt. School of Delhi. International Journal of Nursing Education. 2017; 9(3):132-137. doi: 10.5958/0974-9357.2017.00083.6. 28. Bhagya S, Shanmugaraju P. Effectiveness of Structured Teaching Programme on Knowledge Regarding Preventive Measures of Oral Cancer among Late Adolescents in Selected Junior College, Karminagar, Telangana. Asian J. Nursing Edu. and Research. 2017; 7(1):21-25. doi: 10.5958/23492996.2017.00006.4.

29. Gopi D, Deepa S. Effectiveness of Structured Teaching Programme on Knowledge and Attitude towards Alcohol Abuse among Adolescent Boys. Asian J. Nur. Edu. And Research. 2017; 7(2):173176. doi: $10.5958 / 2349$ -

2996.2017.00035.0. 
30. Beena MR. Effect of Instructional Programme on Knowledge of Adolescent Girls Regarding Reproductive Health. International Journal of Nursing Education. 2016; 8(3):68-71. doi: 10.5958/0974-9357.2016.00093.3. 31. Jaicy SR, Vathsala S. Effectiveness of Adolescent Reproductive Health Education Programme Using Nurse-Led Teacher Delivered Approach on Knowledge and Attitude of School Going Adolescent Girls In Kerala. Journal of Nursing Education. 2015; 7(3):80-85. doi: 10.5958/0974-9357.2015.00139.7. 32. Mendonca L. Effectiveness of Information Education Communication (IEC) Package on Life Style Practices of Adolescents - A Pilot Study. International Journal of Nursing Education 2014; 6(1):69-72. doi: 10.5958/j.09749357.6.1.014.

33. Kole U, Anuchitra S. A study to evaluate the effectiveness of planned teaching programme on impact of early marriage, pregnancy among adolescent girls in selected rural high schools of Belgaum, Karnataka. Asian J. Nur. Edu. \& Research 2014 [citado em 201805 de mai]; 4(1):61-69. Available from:

http://ajner.com/HTMLPaper.aspx?Journal =Asian $\% 20$ Journal\%20of\%20Nursing $\% 20$ Education\%20and\%20Research;PID=201 4-4-1-13

34. Brinkman SA, Johnson SE, Codde JP, Hart MB, Straton JA, Mittinty MN, Silburn SR. Efficacy of infant simulator programmes to prevent teenage pregnancy: a school-based cluster randomised controlled trial in Western Australia. Lancet. 2016; 388: 2264-2271. doi: 10.1016/S0140-6736(16)30384-1. 35. Smith JJ, Morgan PJ, Plotnikoff RC, Dally KA, Salmon J, Okely AD, Finn TL, Lubans DR. Smart-Phone Obesity Prevention Trial for Adolescent Boys in Low-Income Communities: The ATLAS RCT. Pediatrics. 2014 [citado em 201812 de jun]; 134(3):106-119. Available from: https://pediatrics.aappublications.org/cont ent/134/3/e723

36. Lieshout S, Mevissen F, Waal E, Kok G. Long Live Love+: evaluation of the implementation of an online school-based sexuality education program in the Netherlands. Health Education Research.
2017; 32(3):244-257. doi:

10.1093/her/cyx041.

37. Stege HA, Hilberink SR, Bakker E, Staa ALV. Using a Board Game About Sexual Health with Young People with Chronic Conditions in Daily Practice: A Research into Facilitating and Impeding Factors. Sex Disabil. 2016; V.34:349-361. doi: 10.1007/s11195-016-9448-6. 38. Wong ML, Ng JYS, Chan RKW, Chio MTW, Lim RBT, Koh D. Randomized controlled trial of abstinence and safer sex intervention for adolescents in Singapore: 6-month follow-up. Health Education Research. 2017; 32(3):233-243. doi: 10.1093/her/cyx040.

39. Chan A, Brown B, Sepulveda E, Teran-Clayton L. Evaluation of fotonovela to increase human papillomavirus vaccine knowledge, attitudes, and intentions in a low-income Hispanic community. BMC Res Notes. 2015; 8(615):1-10. doi: 10.1186/s13104-015-1609-7.

40. Aventin A, Lohan M, O'halloran P, Henderson M. Design and development of a film-based intervention about teenage men and unintended pregnancy: Applying the Medical Research Council framework in practice. Evaluation and Program Planning. 2015; 49:19-30. doi: 10.1016/j.evalprogplan.2014.11.003. 41. Fraticelli $F$, Marchetti D, Polcini $F$, Mohn AA, Chiarelli F, Fulcheri M, Vitacolonna E. Technology-based intervention for healthy lifestyle promotion in Italian adolescents. Ann Ist Super Sanità. 2016; 52(1):123-127. doi: 10.4415/ANN_16_01_20.

42. Celis ESP, Smith DD, Castillo MPR, Hurria A, Vivas AMP, Weingarten RG, Mohar A, Guerra YC. Implementation of a School-Based Educational Programto Increase Breast Cancer Awareness and Promote Intergenerational Transmission of Knowledge in a RuralMexican Community. The Oncologis. 2017; 22: 1249-1256. doi:

10.1634/theoncologist.2017-0063. 43. Gaughran M, Asgary, R. On-Site Comprehensive Curriculum to Teach Reproductive Health to Female Adolescents in Kenya. Journal of women's health. 2014; 23(4):358-364. doi: 10.1089/jwh.2013.4523. 
44. Nădășan $V$, Foley $K L$, Pénzes $M$, Paulik E, Mihăicuță S, Ábrám Z, Bálint J, Csibi M, Urbán R. The Short-term Effects of ASPIRA: A Web-based, Multimedia Smoking Prevention Program for Adolescents in Romania: A Cluster Randomized Trial. Nicotine \& Tobacco Research. 2017; 19(8):908-915. doi: 10.1093/ntr/ntw308.

45. Palacheewa N, Tiansawad S, Srisuphan W, Whattananarong K, Duffy SA. Development of an Internet-based Smoking Prevention Program for Young Male Adolescents in Thailand. Pacific Rim Int J Nurs Res. 2014 [citado em 201803 de jul]; 18(3):228-243. Available from: https://www.tcithaijo.org/index.php/PRIJNR/article/view/1 5934

46. Lemos ICS, Miranda MLF, Matias LVR, Lédio MF, Alves ACP, Marques SF. Tecnologia educativa para trabalhar a sexualidade de adolescentes no contexto escolar. R. Interd. 2015 [citado em 2018 10 de jun]; 8(3):110-118. Available from: https://revistainterdisciplinar.uninovafapi.e du.br/index.php/revinter/article/view/738/p df 242

47. Santos AS, Viana MCA, Chaves EMC, Bezerra AM, Júnior JG, Tamboril ACR.

Tecnologia educacional baseada em Nola Pender: promoção da saúde do adolescente. Rev enferm UFPE On line 2018; 12(2):582-588. doi: 10.5205/19818963-v12i2a22609p582-588-2018.

48. Lima AC, Bezerra KC, Sousa DM, Rocha JF, Oriá MO. Construção e Validação de cartilha para prevenção da transmissão vertical do HIV. Acta Paul Enferm. 2017; 30(2):181-189. doi: 10.1590/1982-0194201700028.

49. Regis MRS, Schmidlin IOM, Portela $\mathrm{KN}$, Santiago LML. Material didático impresso versus material didático digital: o que dizem os alunos dos cursos semipresenciais do IFCE. Conex. Ci. e Tecnol. 2015; 9(2):65-72. doi: 10.21439/conexoes.v9i2.734.

50. Farias AC. Material impresso e gêneros textuais. Instituto Federal de Santa Catarina. $2^{\mathrm{a}}$ ed. Florianópolis: IFSC; 2013.

51. Silva MG. Impresso ou digital? As práticas de ler textos no papel e na tela.
Entretextos. 2016 [citado em 201818 de out]; 16(2):67-84. Available from:

http://www.uel.br/seer/index.php/entretext os/article/viewFile/18223/20762

52. Massara CL, Murta FLG, Enk MJ, Araújo AD, Modena CM, Carvalho OS. Caracterização de materiais educativos impressos sobre esquistossomose, utilizados para educação em saúde em áreas endêmicas no Brasil. Epidemiol. Serv. Saude. 2016; 25(3):575-584. doi: 10.5123/s1679-49742016000300013.

53. Partelli ANM, Cabral IE. Histórias sobre álcool em comunidade quilombola: metodologia participativa de criaçãovalidação de quadrinhos por adolescentes. Texto Contexto Enferm. 2017; 26(4):1-12. doi: 10.1590/0104-07072017002820017. 


\section{Anexos}

\section{Lista de figuras}

Figura 1: Descritores para os componentes da pergunta norteadora. Crato, Brasil, 2018.

\begin{tabular}{|l|l|l|l|}
\hline & P (População) & V (Variáveis) & O (Desfecho) \\
\hline Decs & Adolescente & Tecnologia Educacional & Educação em Saúde \\
\hline MeSh & Adolescent & Educational Technology & Health Education \\
\hline
\end{tabular}

Fonte: Arquivo original da pesquisa.

Figura 2: Fluxograma do processo de identificação, seleção, elegibilidade e inclusão dos estudos, elaborado a partir da recomendação do fluxograma PRISMA ${ }^{8}$. Crato, Brasil, 2018.

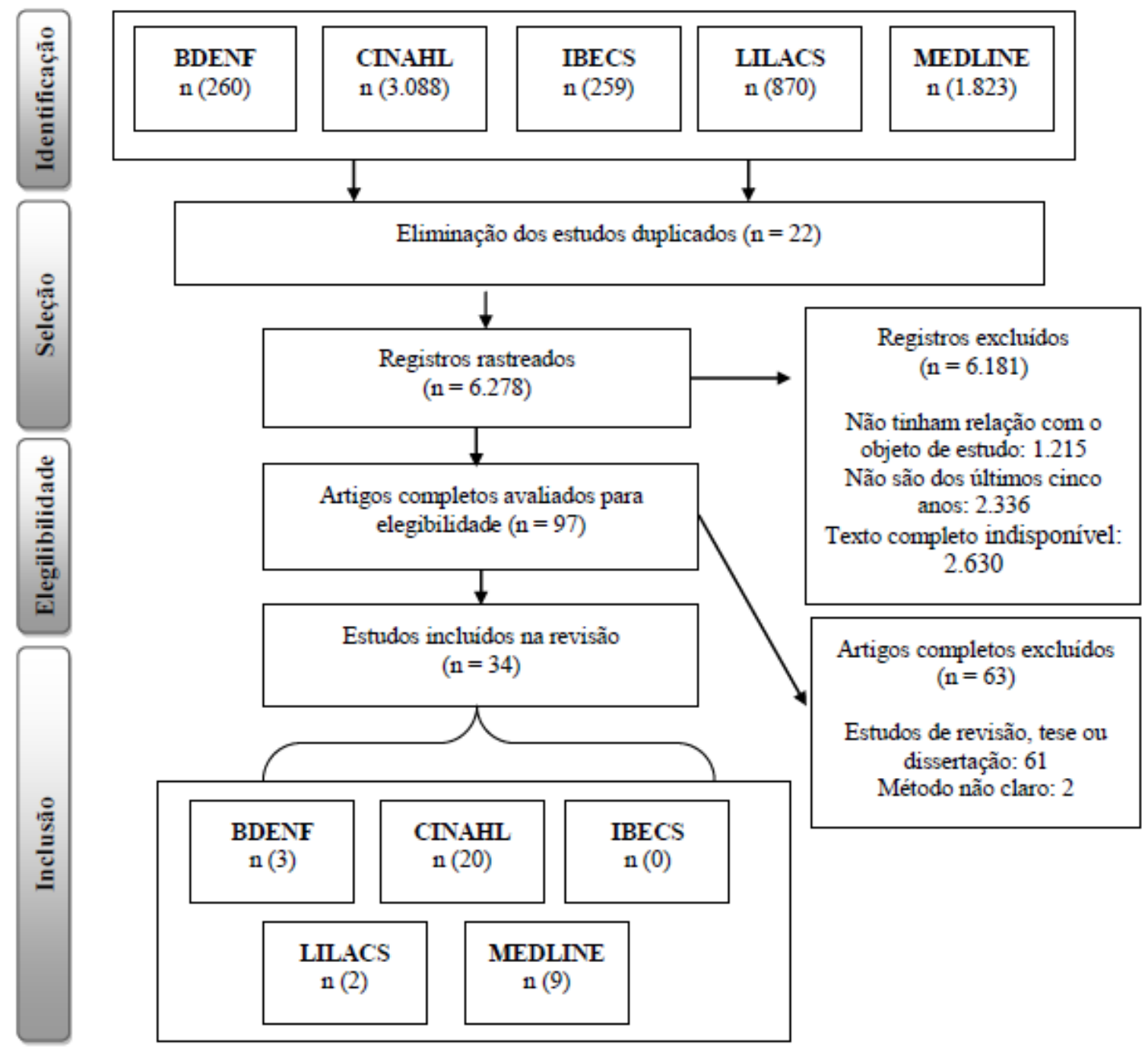

Fonte: Arquivo original da pesquisa.

Rev. Saúde Digital Tec. Educ., Fortaleza, CE, v. 5, n. 1, p.66-82, jan./abr. 2020.

ISSN: 2525-9063 


\section{Lista de quadros}

Quadro 1: Descrição dos estudos inclusos na revisão integrativa. Crato-CE, Brasil, 2018.

\begin{tabular}{|c|c|c|c|}
\hline $\begin{array}{l}\text { Autores e } \\
\text { Países }\end{array}$ & $\begin{array}{l}\text { Tipos de estudo e } \\
\text { níveis de evidência }\end{array}$ & Tecnologia educacional & Implicações para prática \\
\hline $\begin{array}{l}\text { Sousa et al., } \\
\text { 2018/Brasil }\end{array}$ & $\begin{array}{l}\text { Estudo } \\
\text { metodológico/(6) }\end{array}$ & $\begin{array}{lr}\text { Jogo educativo } & \text { sobre } \\
\text { sexualidade } & \text { para } \\
\text { adolescentes. } & \end{array}$ & $\begin{array}{l}\text { Material confiável na prática } \\
\text { educativa com adolescentes. }\end{array}$ \\
\hline $\begin{array}{l}\text { Gonçalves et } \\
\text { al., 2018/Brasil }\end{array}$ & $\begin{array}{lr}\text { Estudo } & \text { quantitativo, } \\
\text { ensaio } & \text { não } \\
\text { controlado/(3) }\end{array}$ & $\begin{array}{l}\text { Educação } \quad \text { em saúde pelo } \\
\text { facebook } \quad \text { (sobrepeso } \\
\text { lobesidade e satisfação com } \\
\text { a imagem corporal). }\end{array}$ & $\begin{array}{l}\text { Não houve impacto estatisticamente } \\
\text { significativo da estratégia de } \\
\text { educação em saúde implementada. }\end{array}$ \\
\hline $\begin{array}{l}\text { Widman et al., } \\
\text { 2018/EUA }\end{array}$ & $\begin{array}{l}\text { Estudo } \\
\text { randomizado/(2) }\end{array}$ & $\begin{array}{l}\text { Programa de saúde sexual } \\
\text { on-line. }\end{array}$ & $\begin{array}{l}\text { Nova opção na crescente gama de } \\
\text { intervenções em saúde digital. }\end{array}$ \\
\hline $\begin{array}{l}\text { Oman et al., } \\
2018 / \text { EUA }\end{array}$ & $\begin{array}{l}\text { Ensaio controlado } \\
\text { randomizado/(2) }\end{array}$ & $\begin{array}{l}\text { Programa de prevenção da } \\
\text { gravidez na adolescência. }\end{array}$ & $\begin{array}{l}\text { Intervenção eficaz de educação em } \\
\text { saúde sexual. }\end{array}$ \\
\hline $\begin{array}{l}\text { Silva et al., } \\
\text { 2017/Brasil }\end{array}$ & $\begin{array}{l}\text { Estudo } \\
\text { metodológico/(6) }\end{array}$ & $\begin{array}{lr}\text { Jogo educativo } & \text { para } \\
\text { adolescentes } & \text { sobre } \\
\text { amamentação. } & \\
\end{array}$ & $\begin{array}{l}\text { Elimina dúvidas que permeiam o } \\
\text { cotidiano de mães adolescentes, } \\
\text { incentivando o aleitamento materno. }\end{array}$ \\
\hline $\begin{array}{l}\text { Savita; Ranjitha, } \\
\text { 2017/IIndia }\end{array}$ & $\begin{array}{l}\text { Pesquisa avaliativa } \\
\text { descritiva /(6) }\end{array}$ & $\begin{array}{l}\text { Programa de } \quad \text { ensino } \\
\text { planejado em conhecimentos } \\
\text { e práticas sobre higiene } \\
\text { menstrual. }\end{array}$ & $\begin{array}{l}\text { Diferença significativa } \quad \text { no } \\
\text { conhecimento e na prática em relação } \\
\text { à higiene menstrual. }\end{array}$ \\
\hline $\begin{array}{l}\text { Bhagya; } \\
\text { Shanmuga, } \\
\text { 2017/ Índia }\end{array}$ & $\begin{array}{l}\text { Pesquisa avaliativa } \\
\text { descritiva /(6) }\end{array}$ & $\begin{array}{l}\text { Programa de ensino na } \\
\text { prevenção do câncer bucal } \\
\text { em adolescentes tardios. }\end{array}$ & $\begin{array}{l}\text { Altamente eficaz na melhoria do } \\
\text { conhecimento dos adolescentes } \\
\text { tardios, em relação às medidas } \\
\text { preventivas do câncer bucal. }\end{array}$ \\
\hline $\begin{array}{l}\text { Wong et al., } \\
2017 / \\
\text { Cingapura }\end{array}$ & $\begin{array}{l}\text { Estudo controlado } \\
\text { randomizado/(2) }\end{array}$ & $\begin{array}{lr}\text { Intervenção } & \text { comportamental } \\
\text { (Flipcharts, } & \text { vídeos, } \\
\text { dramatização e bate-papo } \\
\text { on-line } \\
\text { comportamentos de redução } \\
\text { de risco de infecções } \\
\text { sexualmente transmissíveis). }\end{array}$ & $\begin{array}{l}\text { Efeito significativo sobre a } \\
\text { abstinência em homens jovens e } \\
\text { mantendo um parceiro em mulheres } \\
\text { jovens. }\end{array}$ \\
\hline $\begin{array}{l}\text { Lieshout et al., } \\
\text { 2017/Holanda }\end{array}$ & qualitativo & Website de educação sexual. & $\begin{array}{l}\text { Diversidade nos exercícios e na } \\
\text { relevância do caráter interativo. }\end{array}$ \\
\hline $\begin{array}{l}\text { Celis et al., } \\
\text { 2017/ México }\end{array}$ & Estudo piloto/(6) & $\begin{array}{l}\text { Programa educacional de } \\
\text { câncer de mama, baseado na } \\
\text { escola para adolescentes, em } \\
\text { uma comunidade rural } \\
\text { mexicana. }\end{array}$ & $\begin{array}{l}\text { Aumentou o conhecimento dos } \\
\text { adolescentes sobre o câncer de } \\
\text { mama e promoveu a transmissão } \\
\text { intergeracional desse conhecimento } \\
\text { para familiares. }\end{array}$ \\
\hline $\begin{array}{l}\text { Souza et al., } \\
\text { 2016/Brasil }\end{array}$ & Estudo analítico/(6) & Jogo on-line "Papo Reto". & $\begin{array}{l}\text { Possibilitou que os adolescentes se } \\
\text { tornassem criativos e ativos na } \\
\text { produção de sentidos, na criação de } \\
\text { discursos e nas formas de pensar, } \\
\text { sentir e agir no campo da } \\
\text { sexualidade. }\end{array}$ \\
\hline $\begin{array}{l}\text { Gopi; Deepa, } \\
\text { 2016/Índia }\end{array}$ & $\begin{array}{l}\text { Estudo pré- } \\
\text { experimental/(3) }\end{array}$ & $\begin{array}{l}\text { Programa de ensino } \\
\text { estruturado sobre } \quad 0 \\
\text { conhecimento e a atitude em } \\
\text { relação ao abuso de álcool } \\
\text { entre meninos adolescentes } \\
\text { escolares. }\end{array}$ & $\begin{array}{l}\text { Ajudou o aluno a adquirir } \\
\text { conhecimento e aumentar a atitude } \\
\text { em relação à prevenção do abuso de } \\
\text { álcool. }\end{array}$ \\
\hline
\end{tabular}




\begin{tabular}{|c|c|c|c|}
\hline $\begin{array}{l}\text { Beena, } \\
2016 / \text { Índia }\end{array}$ & \begin{tabular}{ll|} 
Estudo & pré- \\
experimental/(3)
\end{tabular} & $\begin{array}{l}\text { Programa de ensino assistido } \\
\text { por vídeo. }\end{array}$ & $\begin{array}{l}\text { Eficaz na melhoria do conhecimento } \\
\text { de meninas adolescentes em relação } \\
\text { à saúde reprodutiva. }\end{array}$ \\
\hline $\begin{array}{l}\text { Francis et al., } \\
\text { 2016/ EUA }\end{array}$ & $\begin{array}{l}\text { Delineamento } \\
\text { randomizado/(2) }\end{array}$ & $\begin{array}{l}\text { Programa de Extensão para } \\
\text { Adolescentes. }\end{array}$ & $\begin{array}{l}\text { Poucas evidências para apoiar a } \\
\text { eficácia do programa na redução de } \\
\text { comportamentos sexuais de risco. }\end{array}$ \\
\hline $\begin{array}{l}\text { Calise et al., } \\
\text { 2016/ EUA }\end{array}$ & $\begin{array}{l}\text { Estudo randomizado } \\
\text { controlado /(2) }\end{array}$ & $\begin{array}{l}\text { Programa } \\
\text { Saudáveis. }\end{array}$ & $\begin{array}{l}\text { Evidência de atraso na iniciação } \\
\text { sexual até o final do programa para } \\
\text { meninas e hispânicos, mas não para } \\
\text { meninos. }\end{array}$ \\
\hline $\begin{array}{l}\text { Stege et al., } \\
2016 / \text { Holanda }\end{array}$ & $\begin{array}{l}\text { Estudo transversal } \\
\text { de métodos mistos } \\
/(6)\end{array}$ & $\begin{array}{l}\text { Jogo de tabuleiro sobre } \\
\text { saúde sexual. }\end{array}$ & $\begin{array}{l}\text { Atitude positiva em relação à } \\
\text { discussão da sexualidade. }\end{array}$ \\
\hline $\begin{array}{l}\text { Wang; Singhal, } \\
\text { 2016/EUA }\end{array}$ & $\begin{array}{l}\text { Abordagem de } \\
\text { métodos mistos } /(6)\end{array}$ & $\begin{array}{l}\text { Mensagens educativas em } \\
\text { narrativas de entretenimento } \\
\text { em plataformas digitais. }\end{array}$ & $\begin{array}{l}\text { Amplo alcance de público, potencial } \\
\text { envolvimento dos espectadores e } \\
\text { impacto cognitivo, emocional e social } \\
\text { na comunicação e educação } \\
\text { em saúde sexual e reprodutiva. }\end{array}$ \\
\hline $\begin{array}{l}\text { Nădășan et al., } \\
\text { 2016/Romênia }\end{array}$ & $\begin{array}{l}\text { Ensaio controlado } \\
\text { aleatorizado /(3) }\end{array}$ & $\begin{array}{l}\text { Programa de multimídia para } \\
\text { prevenção de tabagismo. }\end{array}$ & $\begin{array}{l}\text { Pode diminuir a iniciação do } \\
\text { tabagismo entre adolescentes } \\
\text { multiétnicos na Europa Central e } \\
\text { Oriental. }\end{array}$ \\
\hline $\begin{array}{l}\text { Brinkman et al., } \\
\text { 2016/Austrália }\end{array}$ & $\begin{array}{l}\text { Ensaio clínico } \\
\text { randomizado } \\
\text { controlado/(2) }\end{array}$ & Programa virtual. & $\begin{array}{l}\text { O programa baseado em simuladores } \\
\text { infantis não atingiu o objetivo de } \\
\text { reduzir a gravidez na adolescência. }\end{array}$ \\
\hline $\begin{array}{l}\text { Fraticelli et al., } \\
\text { 2016/França }\end{array}$ & $\begin{array}{l}\text { Estudo } \\
\text { quantitativo/(6) }\end{array}$ & Jogo virtual. & $\begin{array}{l}\text { Propriedade de entretenimento para } \\
\text { promover estilo de vida saudável } \\
\text { entre adolescentes italianos. }\end{array}$ \\
\hline $\begin{array}{l}\text { Silva et al., } \\
\text { 2015/Brasil }\end{array}$ & $\begin{array}{l}\text { Estudo transversal de } \\
\text { abordagem } \\
\text { quantitativa/(6) }\end{array}$ & $\begin{array}{l}\text { Jogo de labirinto, acerca de } \\
\text { pessoa com deficiência. }\end{array}$ & $\begin{array}{lll}\text { Apreensão } & \text { do } & \text { conhecimento } \\
\text { referente aos tipos de deficiência } \\
\text { abordados. }\end{array}$ \\
\hline $\begin{array}{l}\text { Jaicy; Vathsala, } \\
\text { 2015/Índia }\end{array}$ & $\begin{array}{l}\text { Estudo experimental } \\
/(3)\end{array}$ & $\begin{array}{l}\text { Programa de ensino } \\
\text { saúde rem } \\
\text { adolescência. }\end{array}$ & $\begin{array}{l}\text { O conhecimento e a atitude foram } \\
\text { significativamente aumentados após } \\
\text { a intervenção. }\end{array}$ \\
\hline $\begin{array}{l}\text { Chan et al., } \\
\text { 2015/Espanha }\end{array}$ & $\begin{array}{ll}\text { Estudo } & \text { pré- } \\
\text { experimental/(3) }\end{array}$ & $\begin{array}{lll}\begin{array}{l}\text { Fotonovela } \\
\text { fotografia). }\end{array} & \text { (história } \quad \mathrm{da} \\
\end{array}$ & $\begin{array}{l}\text { Pode ser veículo poderoso para } \\
\text { educação do Papiloma Vírus Humano } \\
\text { (HPV), particularmente entre os } \\
\text { hispânicos, podendo promover } \\
\text { mudanças positivas r no } \\
\text { conhecimento, nas atitudes e nas } \\
\text { intenções. }\end{array}$ \\
\hline $\begin{array}{l}\text { Andrade et al., } \\
\text { 2015/EUA }\end{array}$ & $\begin{array}{l}\text { Estudo metodológico } \\
\text { com abordagem } \\
\text { qualitativa/(6) }\end{array}$ & Webr & $\begin{array}{l}\text { Orienta os jovens a compreender as } \\
\text { escolhas e consequências da vida, } \\
\text { disseminando mensagens de } \\
\text { prevenção, com ênfase ao abuso de } \\
\text { substâncias, risco sexual e violência. }\end{array}$ \\
\hline $\begin{array}{l}\text { Kohlstadt et al., } \\
\text { 2015/EUA. }\end{array}$ & $\begin{array}{ll}\text { Estudo } & \text { de } \\
\text { desenvolvimento/(6) }\end{array}$ & $\begin{array}{l}\text { Plataforma digital para uma } \\
\text { intervenção nutricional. }\end{array}$ & $\begin{array}{l}\text { Os jovens criaram materiais de } \\
\text { intervenção } \\
\text { comportamentos positivos de saúde } \\
\text { on-line. }\end{array}$ \\
\hline $\begin{array}{l}\text { Aventin et al., } \\
2015 / \text { /rlanda }\end{array}$ & $\begin{array}{ll}\text { Estudo } & \text { de } \\
\text { desenvolvimento/(6) }\end{array}$ & Vídeo. & $\begin{array}{l}\text { Influenciam na mudança de } \\
\text { comportamento sexual e incorporam } \\
\text { entendimentos sociológicos das } \\
\text { normas de gênero relacionadas à } \\
\text { gravidez. }\end{array}$ \\
\hline $\begin{array}{l}\text { Smith et al., } \\
\text { 2014/ Austrália }\end{array}$ & $\begin{array}{l}\text { Ensaio controlado } \\
\text { randomizado/(2) }\end{array}$ & $\begin{array}{l}\text { Intervenção } \quad \text { através } \\
\text { Smartphones. }\end{array}$ & $\begin{array}{l}\text { Sem efeitos significativos na } \\
\text { composição corporal, mas bem } \\
\text { sucedida na melhoria da aptidão } \\
\text { muscular, habilidades de movimento }\end{array}$ \\
\hline
\end{tabular}

Rev. Saúde Digital Tec. Educ., Fortaleza, CE, v. 5, n. 1, p.66-82, jan./abr. 2020.

ISSN: 2525-9063 


\begin{tabular}{|c|c|c|c|}
\hline & & & $\begin{array}{l}\text { e comportamentos relacionados com } \\
\text { o peso. }\end{array}$ \\
\hline $\begin{array}{l}\text { Maximino; } \\
\text { Picolini-Pereira; } \\
\text { Carvalho, 2014/ } \\
\text { Brasil }\end{array}$ & $\begin{array}{l}\text { Estudo metodológico } \\
\text { de abordagem } \\
\text { quantitativa/(6) }\end{array}$ & $\begin{array}{l}\text { Programa educacional } \\
\text { (Cybertutor) em síndromes } \\
\text { genéticas para alunos do } \\
\text { ensino fundamental (entre } 13 \\
\text { e } 14 \text { anos). }\end{array}$ & $\begin{array}{l}\text { Pode ser utilizado como ferramenta } \\
\text { para fins educacionais em síndromes } \\
\text { genéticas de outras populações. }\end{array}$ \\
\hline $\begin{array}{l}\text { Palacheewa et } \\
\text { al., 2014/ } \\
\text { Tailândia }\end{array}$ & $\begin{array}{l}\text { Estudo sistemático } \\
\text { de planejamento, } \\
\text { desenvolvimento, } \\
\text { e avaliação/(6) }\end{array}$ & Site. & $\begin{array}{l}\text { Estratégia interativa para melhorar o } \\
\text { conhecimento dos adolescentes, a } \\
\text { atitude e autoeficácia para prevenção } \\
\text { do tabagismo. }\end{array}$ \\
\hline $\begin{array}{l}\text { Mendonça, } \\
\text { 2014/Índia }\end{array}$ & $\begin{array}{l}\text { Estudo } \\
\text { experimental/(3) }\end{array}$ & 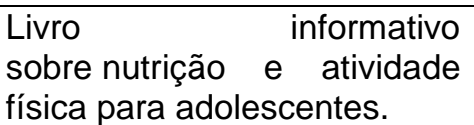 & $\begin{array}{l}\text { Eficaz na melhoria das práticas de } \\
\text { estilo de vida. }\end{array}$ \\
\hline $\begin{array}{l}\text { Kole; Anuchitra, } \\
\text { 2014/Índia }\end{array}$ & $\begin{array}{l}\text { Estudo } \\
\text { experimental/(3) }\end{array}$ & $\begin{array}{l}\text { Programa de ensino } \\
\text { planejado. }\end{array}$ & $\begin{array}{l}\text { Ganho significativo no conhecimento } \\
\text { do grupo experimental após o } \\
\text { planejamento do programa de ensino } \\
\text { sobre o impacto do casamento } \\
\text { precoce e gravidez. }\end{array}$ \\
\hline $\begin{array}{l}\text { Katz et al., } \\
2014 / \text { EUA }\end{array}$ & $\begin{array}{lr}\text { Estudo } & \text { de } \\
\text { desenvolvimento } & \mathrm{e} \\
\text { teste piloto } & \text { de } \\
\text { aceitabilidade/(6) } & \end{array}$ & $\begin{array}{l}\text { História em quadrinhos } \\
\text { (HQ) sobre a vacina contra } \\
0 \quad \text { HPV para } \\
\text { adolescentes. }\end{array}$ & $\begin{array}{l}\text { Conteúdo aceitável, fácil de ler e uma } \\
\text { boa maneira de aprender sobre ser } \\
\text { saudável. }\end{array}$ \\
\hline $\begin{array}{l}\text { DiClemente et } \\
\text { al., 2014/ EUA }\end{array}$ & $\begin{array}{l}\text { Ensaio clínico } \\
\text { randomizado/(2) }\end{array}$ & $\begin{array}{l}\text { Intervenção por telefone para } \\
\text { sustentar comportamentos } \\
\text { preventivos de IST e HIV. }\end{array}$ & $\begin{array}{l}\text { A sustentação do impacto, a longo } \\
\text { prazo, de uma intervenção IST/HIV é } \\
\text { possível, com o aconselhamento } \\
\text { telefónico breve e personalizado. }\end{array}$ \\
\hline $\begin{array}{l}\text { Gaughran; } \\
\text { Asgary, } \\
\text { 2014/Quênia }\end{array}$ & $\begin{array}{lr}\text { Estudo } & \text { metodológico } \\
\text { com } & \text { abordagem } \\
\text { mista/(6) } & \\
\end{array}$ & $\begin{array}{l}\text { Utilização de jogos } \\
\text { educativos na educação em } \\
\text { saúde reprodutiva. }\end{array}$ & $\begin{array}{l}\text { Viável, bem recebido e atingiu os } \\
\text { objetivos educacionais. }\end{array}$ \\
\hline
\end{tabular}

Nota: (2) nível 2; (3) nível 3; (6) nível 6.

Fonte: Arquivo original da pesquisa. 


\section{Como citar este artigo}

Silva MY, Gonçalves, DE, Martins, AKL. Tecnologias educacionais como estratégia para educação em saúde dos adolescentes: revisão integrativa. Revista de Saúde Digital e Tecnologias Educacionais. [online], volume 5, n. 1. Editor responsável: Luiz Roberto de Oliveira. Fortaleza, mês e ano, p. 67-82. Disponível em: http://periodicos.ufc.br/resdite/index. Acesso em "dia/mês/ano".

Data de recebimento do artigo: 16/10/2019

Data de aprovação do artigo: 05/02/2020

Data de publicação: 17/04/2020 